In: Program and abstracts of the 9th International Conference on AIDS (Berlin). Berlin: Institute for Clinical and Experimental Virology of the Free University of Berlin, 1993; 9:304.

2. Swerdlow DL, Ries AA. Cholera in the Americas - guidelines for the clinician. JAMA 1992;267:1495-99.

\section{Pneumonia and Respiratory Distress Syndrome During Primary Infection with Toxoplasma gondii}

SIR-A 26-year-old male acquired human immunodeficiency virus (HIV) infection through homosexual contact in 1987. He received therapy with trimethoprim-sulfamethoxazole (TMP-SMZ; 160/800 mg three times per week) starting in November 1992 and acquired a kitten in the fall of 1992. Tests for toxoplasmal antibodies (IMX Toxo IgG and IMX Toxo IgM; Abbott Laboratories, Abbott Park, IL) were negative in January 1991, March 1992, October 1992, and September 1993.

In October 1993 the patient started complaining of a dry cough. Serologies for antibody to Toxoplasma gondii were again negative. He was admitted to the hospital on 1 December 1993 for fever (temperature, $40^{\circ} \mathrm{C}$ ), cough, and a bilateral interstitial lung infiltrate.

Laboratory findings on admission included moderate pancytopenia, an elevated level of lactate dehydrogenase $(6,450 \mathrm{U} / \mathrm{L}$; normal range, 187-443 U/L), and an elevated level of creatine kinase $(2,853 \mathrm{U} / \mathrm{L}$; normal range, $0-270)$. The CD4 cell count was $54 / \mu \mathrm{L}$. A chest roentgenogram showed bilateral reticulonodular infiltrates with alveolar exudates and mediastinal adenopathies. Auraminerhodamine and Ziehl-Neelsen stains of bronchoalveolar lavage fluid were negative, as were cultures for bacteria and atypical mycobacteria, but multiple $T$. gondii tachyzoites were seen on gram staining and acridine orange staining (figure 1). Serologic tests for IgG and IgM antibodies to $T$. gondii were positive.

The patient started receiving therapy with high-dose sulfadiazine $(6 \mathrm{~g} / \mathrm{d} \mathrm{iv})$ and pyrimethamine $(200 \mathrm{mg} / \mathrm{d}$ followed by $75 \mathrm{mg} / \mathrm{d} \mathrm{iv})$ plus prednisone $(60 \mathrm{mg} / \mathrm{d}$ po; dose was tapered over the next 2 weeks) and folinic acid. His condition improved rapidly, and he was still alive in October 1994; at that time he did not have fever or pulmonary symptoms and he was receiving $25 \mathrm{mg}$ of pyrimethamine and $2 \mathrm{~g}$ of sulfadiazine per day.

Disseminated toxoplasmosis in association with lung involvement and acute respiratory distress syndrome is rare [1]. Examination of bronchoalveolar fluid after Giemsa or gram staining is an effective method of diagnosing this condition [2]. Extremely elevated levels of lactate dehydrogenase, as occurred in our case, are typical and help to differentiate pulmonary toxoplasmosis from Pneumocystis carinii pneumonia [3].

Reprints or correspondence: Dr. Bernard Hirschel, Division of Infectious Diseases, Hôpital Cantonal Universitaire, CH-1211 Geneva 14, Switzerland.

Clinical Infectious Diseases 1995;21:690-1

(C) 1995 by The University of Chicago. All rights reserved. $1058-4838 / 95 / 2103-0040 \$ 02.00$
3. Arathoon E, Estrada y Martin RM, Mayorga R. HIV-seroprevalence survey in a Guatemalan AIDS clinic [abstract no PO BO4-1009]. In: Program and abstracts of the 9th International Conference on AIDS (Berlin). Berlin: Institute for Clinical and Experimental Virology of the Free University of Berlin, 1993;9:303.

Pulmonary toxoplasmosis usually represents reactivation of quiescent parasitic infection after immunosuppression because most patients have toxoplasmal antibodies before pulmonary symptoms develop [1]. The course of primary toxoplasmosis in immunosuppressed patients is unknown, although case reports of transmission by transfusion into patients with leukemia have suggested that it may be life threatening [4].

Our patient had clinical and radiological features that were typical of toxoplasmal pneumonia. The diagnosis was made by examination of bronchoalveolar lavage fluid. The strong circumstantial evidence that our patient had primary toxoplasmal infection is as follows: he had been exposed to a cat that he had recently acquired, no IgG antibodies were detected during the 2 years before admission, and seroconversion occurred (IgG, IgM, and IgA antibodies were detected) during and after clinical disease.

Although in a few cases described in the literature the patients did not have toxoplasmal antibodies at the time of diagnosis of toxoplasmal pneumonia, it has never been clear whether those patients had a reactivation of a previous infection or a newly acquired toxoplasmal infection [1]. Indeed, in the absence of the results of previous serologic tests, it is difficult to assess the presence or absence of latent infection once immunosuppression is extreme [5].

It is interesting to note that TMP-SMZ (160/800 mg three times per week) was administered to our patient in the months before disseminated toxoplasmosis occurred, although we cannot be assured that the patient complied with this regimen. TMP-SMZ is recognized as efficacious prophylaxis against reactivation of toxoplasmosis in patients who are infected with HIV. It is conceivable that these doses are more likely to fail for immunosuppressed patients with primary exposure to $T$. gondii.

Catherine Renold, Joëlle Wintsch, Ingeborg Filthuth, Werner Wunderli, Daniel Lew, and Bernard Hirschel Division of Infectious Diseases, Hopital Cantonal Universitaire, Geneva, Switzerland

\section{References}

1. Pomeroy C, Filice GA. Pulmonary toxoplasmosis: a review. Clin Infect Dis 1992; 14:863-70.

2. Jacobs F, Depierreux M, Goldman M, et al. Role of bronchoalveolar lavage in diagnosis of disseminated toxoplasmosis. Rev Infect Dis 1991; 13:637-41.

3. Pugin J, Vanhems P, Hirschel B, Chave JP, Flepp M. Extreme elevations of serum lactic dehydrogenase differentiating pulmonary toxoplasmosis from pneumocystis pneumonia [letter]. N Engl J Med 1992;326:1226.

4. Siegel SE, Lunde MN, Gelderman AH, et al. Transmission of toxoplasmosis by leukocyte transfusion. Blood 1971;37:388-94.

5. Renold C, Sugar A, Chave JP, et al. Toxoplasma encephalitis in patients with the acquired immunodeficiency syndrome. Medicine (Baltimore) 1992:71:224-39. 
Figure 1. Tachyzoites of Toxoplasma gondii (arrows) in bronchial lavage fluid from an HIV-infected patient with pneumonia and respiratory distress syndrome. Top, gram stain; bottom, acridine orange stain (magnification, $\times 600$ ).

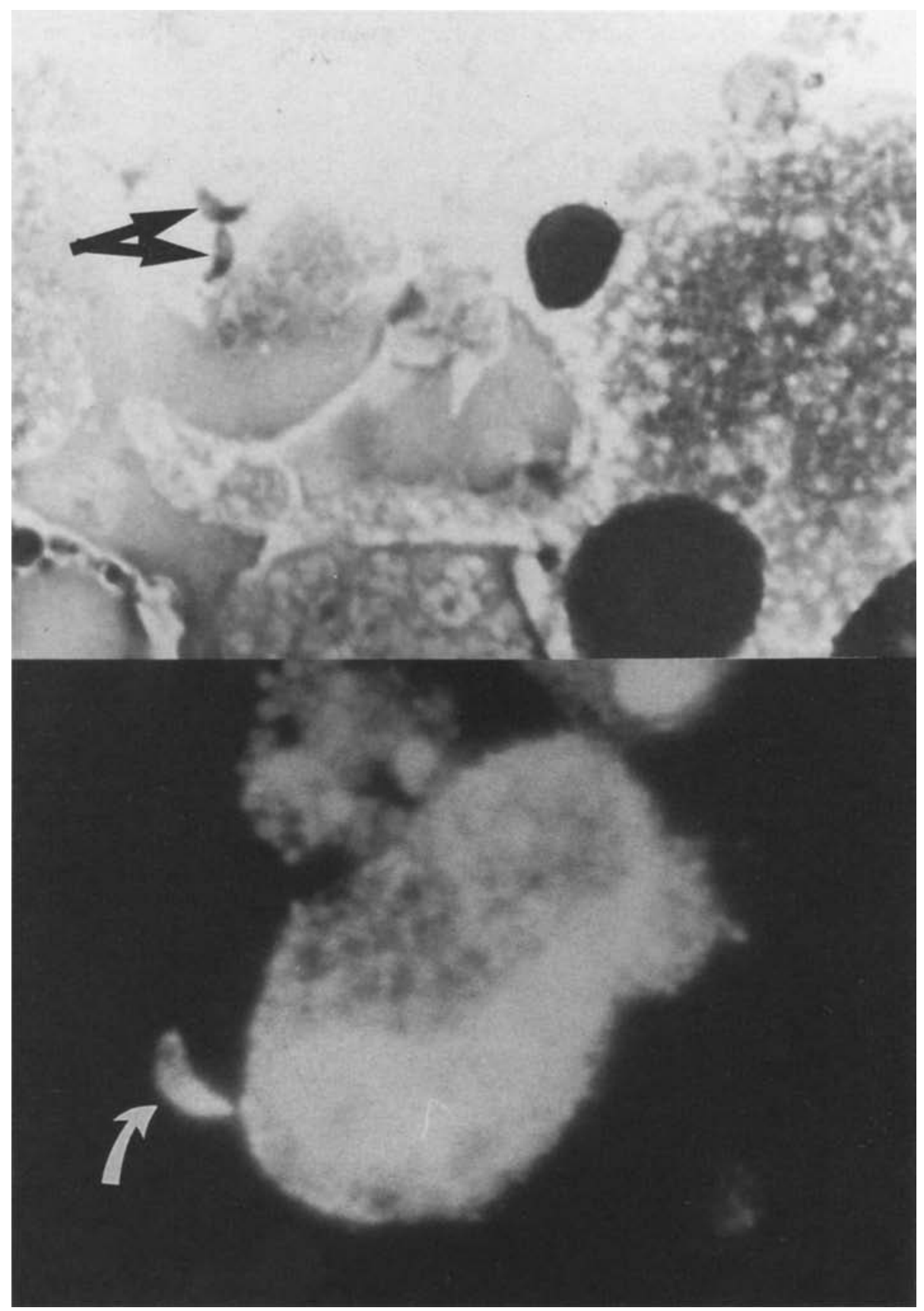

